\title{
Experimental investigation on creep behavior of clastic rock
}

\author{
Zhang $\mathrm{Yu}^{1,2, *}$, Zhang $\mathrm{Yan}^{2}$, Mei Song-hua ${ }^{1}$ \\ ${ }^{1}$ Hunan Provincial Key Laboratory of Key Technology on Hydropower Development, Zhongnan Engineering Corporation, Changsha \\ 410014, China \\ ${ }^{2}$ College of Pipeline and Civil Engineering, China University of Petroleum, Qingdao, Shandong 266580, China
}

\begin{abstract}
Many deflected fault zones exist under the dam foundation Xiang-jiaba Hydropower Station in southwestern China. Clastic rock is the main medium with poor physical and mechanical properties. In order to study the creep properties of the clastic rock, triaxial compression creep experiments were carried out on a rock servo-controlling rheology testing machine. From the test results, it can be concluded that the clastic rock has obvious creep characteristics, and the time-dependent deformation is large. Based on the test results, the relationship between axial strain and time under different confining pressures is studied. The relationship between axial strain rates and deviatoric stress under different stress levels is also discussed in detail. Furthermore, the creep failure mechanism under different confining pressures is analyzed as well. Therefore, the creep law of the clastic rock specimen is gained. The relationship between the Burgers creep model and its parameters is obtained by fitting the creep curve with Burgers creep model. The result shows that Burgers model can accurately describe the creep properties of the clastic rock in Xiang-jiaba Hydropower Project.
\end{abstract}

\section{Introduction}

The time-dependent behaviors of rock refer to the continuous deformation of rock under constant stress, including deformation, slip and failure. It is one of the most significant mechanical properties of the rocks [1-2]. The most common method to study the mechanical properties of rock is laboratory test. It is of great significance to analyze the creep constitutive relation and parameters of rock to evaluate the long-term stability of rock engineering. At present, many achievements have been made in the experimental study of time-dependent behavior of different rocks at home and abroad [3-8]. However, there are few experimental data reports on the study of rock creep mechanical properties in Hydropower stations and other projects.

This work focuses on the creep characteristics of the clastic rock mined from fractured zone in the dam foundation of Xiangjiaba Hydropower Project. The Limeiwan fractured zone of deflection crosses beneath the dam foundation and distribution and thickness of the fractured zone are relatively large. When the dam foundation was excavated, the left side of the dam body was exposed. As the main media of deflection zone, clastic rock has poor physical and mechanical properties. Therefore, the creep mechanical behavior directly affects the long-term stability of the dam, which should be studied carefully.

\section{$2 \quad$ Triaxial creep test of clastic rock}

\subsection{Lithologic characteristics of tested rocks}

The clastic rock samples were obtained from the $\mathrm{T}^{3}{ }_{2-6}$ sub-petrofabric in the fractured zone of deflection in cataclastic and clastic shapes (Figure 1). It belongs to poor integrity soft rock with highly weathered and has the characteristics of small specific gravity, medium porosity, loose organizational structure, high water content and pore cementation contact. The average natural density and dry density of the samples are $2.375 \mathrm{~g} / \mathrm{cm}^{3}$ and $2.225 \mathrm{~g} / \mathrm{cm}^{3}$, respectively. The specific gravity is 2.68 , and the average of moisture content and porosity are $6.59 \%$ and $18.23 \%$.

Microscopice test was performed on the clastic rock samples, and the microstructure and mineral composition can be seen in Figure 1. It indicated that the microstructure is extremely complex for the studied rock. And the mineral composition of the samples still retained finegrained structural features of feldspar-quartz sandstone in the original rock. The main mineral composition is quartz (including chalcedony), feldspar (K-feldspar and plagioclase), sericite, chlorite, a small amount of iron compounds and trace minerals. The trace minerals include tourmaline, zircon, phosphorites, zoisite, glauconite, etc. The main chemical constituent is $\mathrm{SiO}_{2}(80.75 \%$ -

\footnotetext{
*Corresponding author's e-mail: zhangyu@upc.edu.cn
} 
$83.52 \%$ ), but it is slightly lower than content of metal oxide.
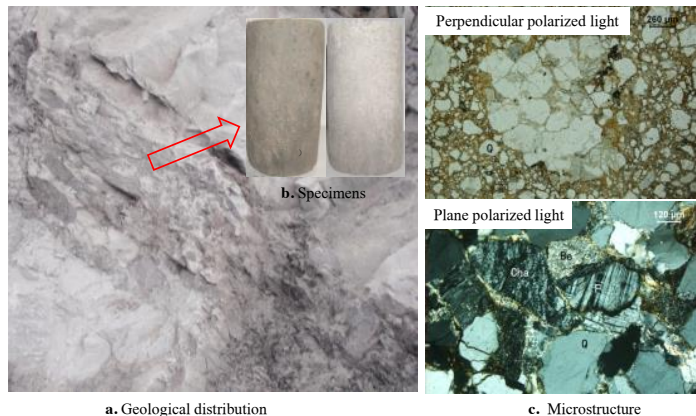

Figure 1 Typical clastic rock in Xiang-jiaba Hydropower Station

\subsection{Experimental equipment}

The test equipment is the rock servo-controlling rheology testing machine developed by Laboratory of Mechanics in Lilleof CNRS in France. This equipment can be used for conventional short-term compression tests and rheological tests. The confining pressure range is $0-60 \mathrm{MPa}$, and the maximum axial pressure is 500MPa. The method of multi-stage loading is used in axial loading, and the stress level is 4-6 levels. Temperature and humidity must be constant during all tests.

\section{Experimental results of triaxial creep}

\subsection{Analysis of creep strain}

The axial strain-time curves at different confining pressure (CP) are shown in Figure 2. The results show that the creep phenomenon of clastic rock is very obvious and the deformation is large with time. The clastic rock exhibits accelerating creep after the last stress loading. At the low deviatoric stress (DS), the creep deformation was not obvious. However, the creep is gradually significant with the increase of the DS. Although the measured values fluctuate, the overall trend tends to be stable.

At the $\mathrm{CP}$ of $1 \mathrm{MPa}$, when the DS level was less than 2.50MPa, the creep deformation was small. When the DS was $45 \mathrm{MPa}$, the axial creep strain increment was $0.71 \mathrm{~mm}$. When the DS reached $3.00 \mathrm{MPa}$, accelerating creep took place after applied stress, and the creep deformation is greater than the previous stress level. After $5 \mathrm{~h}$, the creep deformation increased by $0.83 \mathrm{~mm}$, and finally the rock specimen failed with a large viscous deformation.

At the CP of $2 \mathrm{MPa}$, when the DS was less than $3.25 \mathrm{MPa}$, the creep deformation was unnoticeable. When the stress reaches $4.75 \mathrm{MPa}$, the creep deformation began to accelerate. After 3 hours of dead load, the creep deformation increased by $0.82 \mathrm{~mm}$, and the final failure occurred.

Generally speaking, the confining pressure has a great effect on creep deformation of rock. Under the same conditions, the larger the confining pressure is, the smaller the creep deformation is.

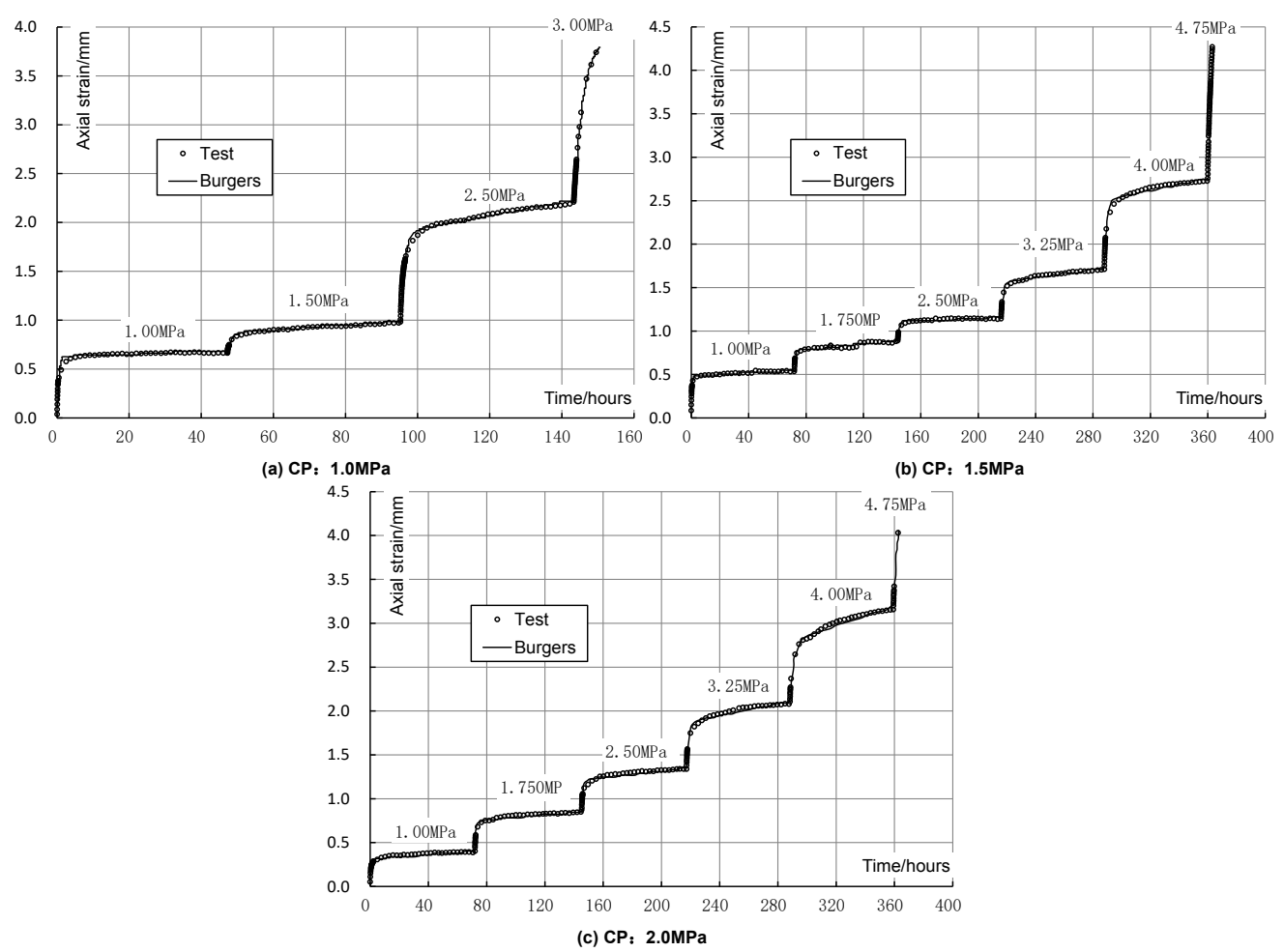

Figure 2 Comparison between Burgers model and creep curves 


\subsection{Analysis on axial creep strain rate}

The creep characteristic of the clastic rock only appears at the primary and steady stage except the final step. Meanwhile, during the creep strain at the low DS, the increase of creep rate gradually decreases to almost zero as time goes on. Under high stress, the characteristics of the creep rate are similar to those under low stress, but the stable creep rate is greater than zero and does not change with time. At the confining pressure of $2 \mathrm{MPa}$, when the stress is $1 \mathrm{MPa}$, the creep rate gradually reduces to a constant value of $0.8 \times 10^{-3} \mathrm{~mm} / \mathrm{h}$. When the stress is $2.5 \mathrm{MPa}$, the stable rheological rate increases to $1.43 \times 10^{-}$ ${ }^{3} \mathrm{~mm} / \mathrm{h}$. When the stress is $4.00 \mathrm{MPa}$, the rate decreases and is kept constant at $5.53 \times 10^{-3} \mathrm{~mm} / \mathrm{h}$. After applying the final stress of $4.75 \mathrm{MPa}$, the creep rate exhibits accelerate stage until the specimen failed eventually, and the process lasted around $3 \mathrm{~h}$. Therefore, the creep rate increased with DS, and the rate first reduced and then remained constant for a period of time. When the load reached final stress, it appeared an accelerating increase.

\subsection{Creep failure mode and mechanism}

The main failure mechanism of such rock was plastic shear with obvious characteristics of compressed and ductility dilatancy. The failure of specimen failure is usually caused by the pore compression and cracks combination. The failure is the result of comprehensive effect of the material defects, heterogeneity and cumulative damage caused by micro-cracks. Based on the micromeso analysis, the relationship between the internal micro-defects and their shape is discussed. It can be seen from the scanning results of the specimen under the scanning electron microscope (SEM) after creep failure (Figure 3 ) that the microscope failure modes are slightly different at various $\mathrm{CP}$. At low $\mathrm{CP}$, there are more growing cracks, rough fracture and less micro grains. As the $\mathrm{CP}$ increases, the fracture surface is clean with more micro grains, and the porosity was decreased. The reason is that the continuous damage caused by the internal microfissures of the specimen accumulates during the test. And then the cracks, originated from the defects of initial internal void, extended and interpenetrated and eventually led to failure. It is the same as macroscopic failure of specimen.

\section{$4 \quad$ Creep model and parameter identification}

The creep curves indicate that the clastic rock specimen has transient deformation under external load. As time goes on, the creep rate decreases gradually and finally reaches a constant value. Furthermore, the specimen exhibits steady creep stage where the rate is more than zero at higher stress level. According to creep characteristics reflected by these curves, Burgers creep model is selected to describe the test. It is regarded as the combination of Maxwell model and Kelvin model. The constitutive equation of Burgers creep model is given as follows:

$$
\left.\begin{array}{l}
\sigma_{\mathrm{M}}=E_{\mathrm{M}} \varepsilon_{\mathrm{M} 1} \\
\sigma_{\mathrm{M}}=\eta_{\mathrm{M}} \dot{\varepsilon}_{\mathrm{M} 2} \\
\sigma_{\mathrm{K}}=E_{\mathrm{K}} \varepsilon_{\mathrm{K}}+\eta_{\mathrm{K}} \dot{\varepsilon}_{\mathrm{K}} \\
\sigma=\sigma_{\mathrm{M}}=\sigma_{\mathrm{K}} \\
\varepsilon=\varepsilon_{\mathrm{M}}+\varepsilon_{\mathrm{K}}
\end{array}\right\}
$$
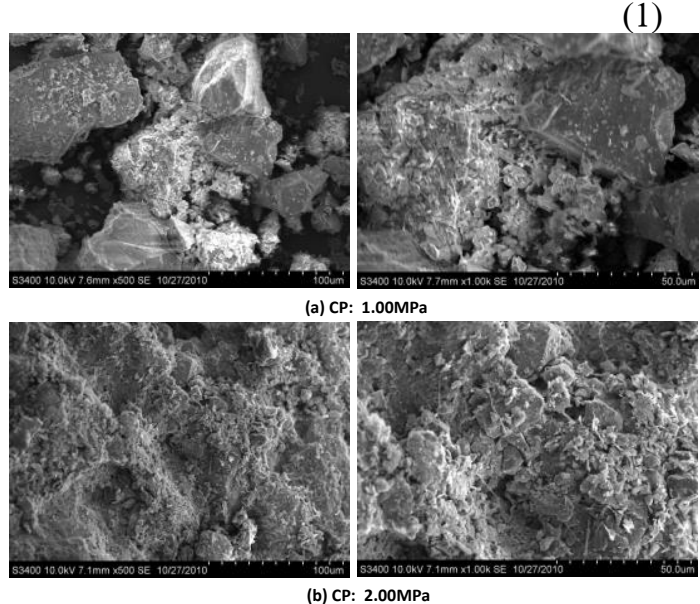

Figure 3 SEM observations for specimen after creep failure

Through Laplace transformation of Eq.(1), the corresponding creep constitutive equation can be obtained:

$$
\varepsilon=\frac{\sigma}{E_{\mathrm{M}}}+\left(\frac{\sigma}{\eta_{\mathrm{M}}}\right) t+\frac{\sigma}{E_{\mathrm{K}}}\left(1-\mathrm{e}^{-\frac{E_{\mathrm{K}}}{\eta_{\mathrm{K}}}}\right)
$$

Within the equation, the subscript "M" represents the mechanical part of Maxwell body as "K" of Kelvin body. $E_{\mathrm{M}}$ and $\eta_{\mathrm{M}}$ are the elastic modulus and viscosity coefficient of Maxwell body as $E_{\mathrm{K}}$ and $\eta_{\mathrm{K}}$ those of Kelvin body.

Based on the results of multi-stage loading test, the Boltzmann superposition method is used to process the test data. Using the least square method based on QuasiNewton search method, the parameters of the Burgers creep model are obtained from the processed data. According to the data sequence $(\varepsilon, t)$ and the Ep.(2), the sum of the squares of the residuals $Q$ can be obtained, which is only the objective function of the least square method.

To determine the parameters, an iteration procedure is used. By using this approach, the fitting curves of the creep model can meet the accuracy requirements. The creep mechanical parameters at different DS and CP are listed in Tables 1 . At the CP of $1.5 \mathrm{MPa}$ and $2.0 \mathrm{MPa}$, the value of the elastic modulus is smaller at the first stress level, which is due the obvious pressure consolidation at the beginning. Although the DS level increases, the elastic modulus remained unchanged. In this stage, the deformation characteristic of rock is linear elastic. When 
the stress continued to increase and reached final stress, the elastic modulus began to decrease rapidly. And the rock sample gradually went into the plastic phase and began to fail. The parameters $\eta_{1}$ represents creep rate of the stable creep. Generally speaking, the rate increases with the stress until it reaches the maximum in the accelerating creep stage. The variation of the rheological pa- rameters $E_{2}$ and $\eta_{2}$ is irregular. The $E_{2} / \eta_{2}$ ratio reflects the length of time from initial creep to steady creep. In addition, the Burgers model can well describe the timedependent behavior of the clastic rock (Figure 2.). In particular, it can accurately simulate the initial creep and steady creep.

Table 1 Creep parameters of clastic rock at various CP

\begin{tabular}{|c|c|c|c|c|c|}
\hline \multirow{2}{*}{$\mathrm{CP} / \mathrm{MPa}$} & \multirow{2}{*}{ Stress level/MPa } & \multicolumn{4}{|c|}{ Burgers model parameters } \\
\hline & & $\mathrm{E}_{1} / \mathrm{GPa}$ & $\mathrm{E}_{2} / \mathrm{GPa}$ & $\eta_{1} /(\mathrm{GPa} * \mathrm{~h})$ & $\eta_{2} /(\mathrm{GPa} * \mathrm{~h})$ \\
\hline \multirow{4}{*}{1.00} & 1.00 & 0.20 & 67.05 & 0.25 & 0.81 \\
\hline & 1.50 & 0.22 & 62.95 & 0.82 & 0.93 \\
\hline & 2.50 & 0.24 & 36.30 & 0.30 & 0.32 \\
\hline & 3.00 & 0.14 & 47.33 & 0.18 & 0.47 \\
\hline \multirow{6}{*}{1.50} & 1.00 & 1.25 & 98.24 & 0.61 & 0.58 \\
\hline & 1.75 & 0.32 & 112.08 & 0.78 & 0.66 \\
\hline & 2.50 & 0.28 & 172.06 & 1.21 & 1.02 \\
\hline & 3.25 & 0.28 & 140.96 & 0.83 & 0.97 \\
\hline & 4.00 & 0.23 & 108.47 & 0.54 & 0.79 \\
\hline & 4.75 & 0.17 & 100.48 & 0.92 & 0.69 \\
\hline \multirow{6}{*}{2.00} & 1.00 & 1.60 & 72.65 & 0.33 & 0.23 \\
\hline & 1.75 & 0.48 & 136.71 & 0.59 & 0.44 \\
\hline & 2.50 & 0.29 & 124.41 & 0.73 & 0.63 \\
\hline & 3.25 & 0.25 & 104.48 & 0.72 & 1.11 \\
\hline & 4.00 & 0.19 & 72.72 & 0.60 & 1.55 \\
\hline & 4.75 & 0.16 & 30.09 & 1.39 & 1.10 \\
\hline
\end{tabular}

\section{Conclusions}

(1) The creep deformation of the clastic rock is not obvious at low DS, but obvious at high axial deviatoric stress level. The time-dependent behavior is very distinct, and the creep deformation is larger. When DS is high, the initial stage and steady stage appear significantly. Creep decreases with an increasing $\mathrm{CP}$, which indicated that the specimen is not easy to creep under high CP.

(2) The creep strain rate of the clastic rock varies at different DS. The creep characteristic of the clastic rock only appears the initial and steady stage except the final step. And the increase of creep rate gradually decreases to nearly zero with time during the creep strain at the low DS. However, the creep rate increased with the increase of the DS. After the final stress is applied, the creep rate exhibits accelerate stages until the specimen failed eventually.

(3) Burgers creep model has a high precision fitting with the measured results. Therefore, the model can overall describe the time-dependent behavior of the clastic rock. It can provide a basis for creep numerical simulation and is of great significance for the prediction of the long term stability of Xiang-jiaba hydropower station.

\section{Acknowledgments}

This work is supported by the Open Research Fund of Hunan Provincial Key Laboratory of Hydropower Development Key Technology (No. PKLHD201601), and supported by CRSRI Open Research Program (CKWV2018475/KY).

\section{References}

1. SUN Jun. (1999) Rheological behavior of geomaterials and its engineering applications. Beijing : China Architecture and Building Press.(in Chinese)

2. SUN Jun. (2007) Rock rheolodical mechanics and its advance in engineering applications. Chinese Journal of Rock Mechanics and Engineering, 26(6): 1081-1106.(in Chinese)

3. HUNSCHE U, SCHULZE O. (1993) Effect of humidity and confining pressure on creep of rock salt. The Mechanical Behavior of Salt of the Third Conference. Germany: Trans. Tech. Publications, 237248 . 
4. MARANINI E, BRIGNOLI M. (1999) Creep behaviour of a weak rock : experimental characterization. Int. J. Rock Mech. Min. Sci., 36(1): 127-138.

5. YANG C H, DAEMEN J J K, YIN J H. (1999) Experimental investigation of creep behavior of salt rock. Int. J. Rock Mech. Min. Sci., 36(2): 233-242.

6. LI Y S, XIA C C. (2000) Time-dependent tests on intact rocks in uniaxial compression. Int. J. Rock Mech. Min. Sci, 37(3): 467-475.

7. FAN Qingzhong, GAO Yanfa. (2005) Experimental study on creep properties of rocks under stepwise loading. Chinese Journal of Geotechnical Engineering, 27(11): 1273-1276.(in Chinese)

8. XU Weiya, YANG Shengqi, YANG Songlin, et al. (2005) Investigation on triaxial rheological mechanical properties of greenschist specimen(I): experimental results.Rock and Soil Mechanics, 26(4): 531537. (in Chinese) 